\title{
ERGODIC TRANSFORMATIONS FROM AN INTERVAL INTO ITSELF $^{1}$ \\ BY
}

TIEN-YIEN LI AND JAMES A. YORKE

\begin{abstract}
A class of piecewise continuous, piecewise $C^{1}$ transformations on the interval $J \subset R$ with finitely many discontinuities $n$ are shown to have at most $n$ invariant measures.
\end{abstract}

1. The way phenomena or processes evolve or change in time is often described by differential equations or difference equations. One of the simplest mathematical situations occurs when the phenomenon can be described by a single number and when this number can be estimated purely as a function of the previous number. That is, when the number $x_{n+1}$ can be written as $x_{n+1}=\tau\left(x_{n}\right)$ where $\tau$ maps an interval $J \subset R$ into itself. For $x \in J$, let $\tau^{0}(x)$ denote $x$ and $\tau^{n+1}(x)$ denote $\tau\left(\tau^{n}(x)\right)$ for $n=0,1, \ldots$ We will say $p \in J$ is a periodic point with period $n$ if $p=\tau^{n}(p)$ and $p \neq \tau^{k}(p)$ for $1 \leqslant k<n$. We say $p$ is a periodic point if $p$ is periodic with some period $n \geqslant 1$. In this paper we assume $\tau$ is piecewise continuous and piecewise twice continuous differentiable. We also assume that

$$
\inf _{x \in J_{1}}\left|\frac{d}{d x} \tau(x)\right|>1 \text { where } J_{1}=\left\{x \in J, \frac{d}{d x} \tau(x) \text { exists }\right\} .
$$

We will refer to the points of $J-J_{1}$ as the points of discontinuity. For such a transformation all periodic points of $\tau$ are unstable. See, for example, [4]. For $x \in J$, let $\Lambda(x)$ be the set of limit points of $\tau^{n}(x)$, that is,

$$
\Lambda(x)=\bigcap_{N=1}^{\infty} \overline{\left\{\tau^{n}(x)\right\}_{n=N}^{\infty}} .
$$

Notice that $\tau(\Lambda(x))=\Lambda(x)$. We show that $\Lambda(x)$ is the union of (one or more) intervals of positive length for almost all $x \in J$. Furthermore, there is a finite collection of sets $L_{1}, \ldots, L_{n}$, where each $L_{i}(i=1, \ldots, n)$ is a union of disjoint intervals, such that for almost all $x \in J, \Lambda(x)$ is one of the sets $L_{i}$.

Received by the editors February 25, 1975 and, in revised form, March 9, 1976.

AMS (MOS) subject classifications (1970). Primary 28A65.

Key words and phrases. Frobenius-Perron operator, invariant measures, function of bounded variation, invariant sets.

${ }^{1}$ Research was partially supported by National Science Foundation under grant MSC 7624432.

- American Mathematical Society 1978 
The intersection $L_{i} \cap L_{j}$ contains at most a finite number of points when $i \neq j$ and each $L_{i}$ contains in its interior a point of discontinuities of $\tau$ and/or $d \tau / d x$. Hence as a corollary we have that if $\tau$ has at most one point of discontinuity then $n=1$ and $\Lambda(x)=\Lambda(y)$ for almost all $x$ and $y$ in $J$.

A measure $\mu$ is said to be invariant (under $\tau$ ) if for all measurable $S \subset J$, we have $\mu(S)=\mu\left(\tau^{-1}(S)\right)$ where $\tau^{-1}(S)=\{x \in J: \tau(x) \in S\}$. In [3], Lasota and Yorke showed for the transformation $\tau$ under study here there exists an absolutely continuous invariant measure. This result generalizes some previous results of Gel'fond [1], Lasota [2], Parry [5] and Rényi [6]. We show in $\$ 2$ that for each $L_{i}$ there exists a unique absolutely continuous invariant measure $\mu_{i}$ which is invariant for $\tau$ such that

$$
\mu_{i}\left(L_{i}\right)=1 \text { and } \mu_{i}\left(L_{j}\right)=0 \text { for } i \neq j .
$$

Furthermore, any absolutely continuously invariant measure $\mu$ can be written as $\Sigma a_{i} \mu_{i}$ for appropriately chosen constants $a_{i}$.

If $\tau$ and $\tau^{\prime}$ have at most one point of discontinuity then there is a unique absolutely continuous invariant measure for $\tau$. Hence $\tau$ is ergodic on $J$. Other properties of these transformations are discussed in [4].

The referee has pointed out that in [8] a similar result is proved using different techniques. They do not estimate the number of invariant measures and so do not discuss conditions under which $\tau$ is ergodic.

2. Let $L^{1}$ denote the space of all integrable functions defined on the interval $[0,1]$ and let $\|\cdot\|$ be the $L^{1}$-norm. Lebesgue measure on $[0,1]$ will be denoted by $m$. We say $f \in L^{1}$ is a function of bounded variation in $L^{1}$ if $f$ equals almost everywhere some function of bounded variation. Let $\tau:[0,1] \rightarrow$ $[0,1]$ be a piecewise continuous and piecewise $c^{2}$-function with $\left\{x_{1}, \ldots, x_{k}\right\}$ $=J-J_{1}$, the points of discontinuity of $\tau$ and $\tau^{\prime}$. We assume (1.1) holds for $\tau$. The Frobenius-Perron operator $P_{\tau}: L^{1} \rightarrow L^{1}$ is defined as a linear operator such that for $f \in L^{1}, P_{\tau} f$ is the function with

$$
\int_{E} P_{\tau} f=\int_{\tau^{-1}(E)} f
$$

for all measurable sets $E$. We say $f$ is invariant (under $\tau$ ) or is an invariant function (of $P_{\tau}$ ) if

$$
\int_{E} f=\int_{\tau^{-1}(E)} f
$$

for every measurable set $E \subset[0,1]$. Notice that $f$ is invariant if and only if $P_{\tau} f=f$ almost everywhere. It is well known that $f$ is invariant under $\tau$ if and only if the measure $d \mu=f d m$ is invariant under $\tau$. In [3], Lasota and Yorke prove that invariant functions of $P_{\tau}$ exist and every invariant function of $P_{\tau}$ is a function of bounded variation in $L^{1}$. 
Definition 2.1. Let $f$ be a function defined on $[0,1]$. We call the set, on which the function $f$ is nonzero, the support of $f$ and denote it, spt $f$. Notice that spt $f$ need not be closed in our definition.

The following property concerning the structure of the support of a bounded variation function is essential for this paper.

Proposition 2.1. If $f$ is a function of bounded variation then

$$
\text { spt } f=\bigcup_{n=0}^{P} K_{n} \cup M, \quad 0 \leqslant P \leqslant \infty,
$$

where $K_{n}$ are disjoint intervals, $M$ is a countable set and

$$
\bigcup_{n=0}^{P} K_{n} \cap M=\varnothing .
$$

Proof. We only need to show that $M$ is at most countable. Suppose this is not the case. Then there exists $m>0$ such that the set $S=\{x: f(x) \geqslant 1 / m\}$ $\cap M$ is uncountable. For, otherwise, the set $M$ would be a union of countable sets. Since $M$ contains no interior points the variation of $f$ over any interval containing $S$ would be unbounded. Hence $M$ is at most countable.

Let $F$ be the set $f \in L^{1}$ which is invariant under $\tau$ (so $F$ is a subspace). As mentioned earlier, by [3], each $f$ in $F$ represents a class of functions which is equal almost everywhere to a function $f_{0}$ of bounded variation. By Proposition 2.1, we can write spt $f_{0}$ as a disjoint union of countable intervals $\left\{K_{n}\right\}$ and a set $M$ which is at most countable. Let $f_{1}=f_{0}$ on $\cup K_{n}$ and $f_{1}=0$ elsewhere. Then, $f_{1}$ equals $f_{0}$ almost everywhere. Hence, we may assume, without loss of generality, that every $f$ in $F$ is a function of bounded variation, and its support consists of closed intervals.

THEOREM 1. There is a finite collection of sets $L_{1}, \ldots, L_{n}$ and a set of functions $\left\{f_{1}, \ldots, f_{n}\right\} \subset F$ such that

(1) each $L_{i}(1<i<n)$ is a finite union of closed intervals;

(2) $L_{i} \cap L_{j}$ contains at most a finite number of points when $i \neq j$;

(3) each $L_{i}(1 \leqslant i \leqslant n)$ contains at least one point of discontinuity $x_{j}$ $(j \in\{1, \ldots, k\})$ in its interior; hence $n \leqslant k$;

(4) $f_{i}(x)=0$ for $x \notin L_{i}, 1<i<n$, and $f_{i}(x)>0$ for almost all $x$ in $L_{i}$;

(5) $\int_{L_{N}} f_{i}(x) d x=1$ for $1 \leqslant i \leqslant n$;

(6) if $g \in F$ satisfies (4) and (5) for some $1 \leqslant i \leqslant n$, then $g=f_{i}$ almost everywhere;

(7) every $f \in F$ can be written as $f=\sum_{i=1}^{n} a_{i} f_{i}$ with suitable chosen $\left\{a_{i}\right\}$.

REMARK. Consider the simple transformation of the form 


$$
\begin{aligned}
& \tau(x)=2 x, \quad 0 \leqslant x \leqslant 1 / 2, \\
& \tau(x)=(2-a)+2(a-1), \quad 1 / 2 \leqslant x \leqslant 1,
\end{aligned}
$$

where $0<a<1 / 2$ (see Figure 1). In [7], S. Ulam pointed out that it was not known even in this simple case whether there was a function invariant under $\tau$. The complete answer to this problem is now clear. In fact, the existence of an invariant function is guaranteed by Theorem 1 of Lasota-Yorke [3] and the uniqueness (up to constant multiples) follows immediately from Theorem 1 stated above.

Before proving Theorem 1, we give the following definitions and a series of lemmas.

Definition 2.2. We write " $A \subset B$ a.e." if $A, B \subset[0,1]$ and $x \in B$ for almost all $x$ in $A$. We write " $A=B$ a.e." if both $A \subset B$ a.e. and $B \subset A$ a.e. are satisfied. We say a set $A$ is invariant (or is invariant under $\tau$ ) if $A$ is a measurable subset of $[0,1]$ and $\tau(A)=A$ a.e. (Notice this does not imply $\tau^{-1}(A)=A$ a.e.)

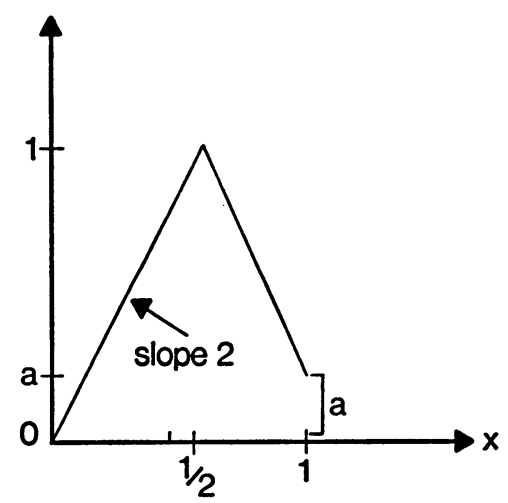

FIGURE 1

We now list some obvious properties of invariant sets which are for later reference. If $A$ is an invariant set, then

$$
\begin{gathered}
m(\tau(A))=m(A), \\
\tau(A) \subset A \quad \text { a.e., }
\end{gathered}
$$

(2.5) for any invariant measure $\mu$ with $d \mu=f d m$ (where $f \in L^{2}$ is invariant), we have

$$
\mu\left(\tau^{-1}(A)-A\right)=0 .
$$

If $A_{1}$ and $A_{2}$ are invariant sets, then

(a) $\tau\left(A_{1} \cap A_{2}\right)=A_{1} \cap A_{2}$ a.e.,

(b) $\tau\left(A_{1} \cup A_{2}\right)=A_{1} \cup A_{2}$ a.e.,

(c) $\tau\left(A_{1}-A_{2}\right)=A_{1}-A_{2}$ a.e. 
These are all quite simple except possibly (2.5) which we obtain as Corollary 2.1 .

LeMma 2.1. Let $A$ be a measurable set satisfying $\tau(A) \subset A$ a.e. Then, for any invariant function $f$, we have

$$
\int_{\tau^{-1}(A)-A} f=0 \text {. }
$$

Proof. Since $\tau(A) \subset A$ a.e., so $A \subset \tau^{-1}(A)$ a.e. Then,

$$
\int_{\tau^{-1}(A)-A} f=\int_{\tau^{-1}(A)} f-\int_{A} f=0 .
$$

Corollary 2.1. If $A$ is invariant, then (2.7) is satisfied.

Write $X_{S}$ for the characteristic function of a set $S \subset[0,1]$.

LEMMA 2.2. Let $A$ be an invariant set. For any invariant function $f$, the product (pointwise multiplication) $f \cdot X_{A}$ is invariant.

Proof. Let $S$ be any measurable set. Then,

$$
\begin{aligned}
\int_{S} f \cdot X_{A} & =\int_{S \cap A} f=\int_{\tau^{-1}(S \cap A)} f=\int_{\tau^{-1}(S) \cap \tau^{-1}(A)} f \\
& =\int_{\tau^{-1}(S) \cap\left(\tau^{-1}(A)-A\right)} f+\int_{\tau^{-1}(S) \cap A} f=\int_{\tau^{-1}(S) \cap A} f .
\end{aligned}
$$

The last equality follows by Corollary 2.1 .

For $f \in L^{2}$, we write $P(f)=\{x: f(x)>0\}$ and $N(f)=\{x: f(x)<0\}$. We will often write $P, N$ for $P(f), N(f)$ when no clarification is needed.

LEMMA 2.3. If $f$ is invariant, the sets $P$ and $N$ are invariant.

Proof. Write

$$
\tau^{-1}(P)=\left(\tau^{-1}(P) \cap P\right) \cup\left(\tau^{-1}(P) \cap P\right) \cup\left(\tau^{-1}(P) \cap Z\right)
$$

where $Z=\{x: f(x)=0\}$. Then,

$$
\int_{P} f=\int_{\tau^{-1}(P)} f=\int_{\tau^{-1}(P) \cap N} f+\int_{\tau^{-1}(P) \cap P} f+\int_{\tau^{-1}(P) \cap Z} f \geqslant \int_{P} f
$$

where the equal sign holds only if

$$
m\left(\tau^{-1}(P) \cap P\right)=m(P) \text { and } m\left(\tau^{-1}(P) \cap N\right)=0 .
$$

That is,

$$
\tau^{-1}(P) \supset P \text { a.e. and } \tau^{-1}(P) \cap N=\varnothing \text { a.e. }
$$

Hence, $\tau(P) \subset P$ a.e. Let $A=\tau(P)$ then $\tau(A) \subset A$ a.e. By Lemma 2.1 


$$
\int_{\tau^{-1}(A)-A} f=0 \text { and } \int_{\tau^{-1}(P)-P} f=0 .
$$

But $P \subset \tau^{-1}(A) \subset \tau^{-1}(P)$ a.e., it follows that

$$
\int_{\tau^{-1}(A)-A} f=\int_{P-\tau(P)} f+\int_{\tau^{-1}(A)-P} f=\int_{P-\tau(P)} f=0 .
$$

Since $f>0$ in $P-\tau(P)$, hence $m(P-\tau(P))-0$. Therefore $\tau(P)=P$ a.e. Similarly $\tau(N)=N$ a.e.

LEMMA 2.4. If $f$ is invariant then $f \cdot X_{P}$ and $f \cdot X_{N}$ are invariant.

Proof. By Lemma 2.2 and Lemma 2.3 these functions are invariant.

Lemmas 2.5 through 2.8 are for demonstrating how to "transform" a set of linearly independent invariant functions into a set of invariant functions with disjoint supports.

LEMMA 2.5. Let $f_{1}$ and $f_{2}$ be invariant and let $S_{1}=\operatorname{spt} f_{1}$ and $S_{2}=\operatorname{spt} f_{2}$. Then,

$$
\begin{gathered}
S_{1} \text { and } S_{2} \text { are invariant, and } \\
S_{1}-S_{1} \cap S_{2} \text { and } S_{2}-S_{1} \cap S_{2} \text { are invariant. }
\end{gathered}
$$

Proof. Since spt $f_{i}=P\left(f_{i}\right) \cup N\left(f_{i}\right)$ for $i=1,2$, by Lemma 2.3 and (2.6) we easily see that both $S_{1}$ and $S_{2}$ are invariant. (2.9) is a direct result of repeated application of (2.6).

LEMMA 2.6. If $f_{1}$ and $f_{2}$ are linearly independent functions in $F$ with $\left\|f_{1}\right\|=$ $\left\|f_{2}\right\|=1$ then there exist $f_{1}^{*}$ and $f_{2}^{*}$ such that

(a) $f_{1}^{*} \geqslant 0, f_{2}^{*} \geqslant 0$ and $\left\|f_{1}^{*}\right\|=\left\|f_{2}^{*}\right\|=1$;

(b) spt $f_{1}^{*}$ and spt $f_{2}^{*}$ are disjoint;

(c) for each $i=1,2$, spt $f_{i}^{*}$ is a union of disjoint intervals contained in spt $f_{1} \cup \operatorname{spt} f_{2}$.

Proof. If $i=1$ or 2 , we have $P\left(f_{i}\right)$ and $N\left(f_{i}\right)$ are both nonempty, then we may let

$$
f_{1}^{*}=\left(f_{i} \wedge 0\right) /\left\|f_{i} \wedge 0\right\| \text { and } f_{2}^{*}=\left(f_{i} \vee 0\right) /\left\|f_{i} \vee 0\right\|
$$

where $f_{i} \vee 0=\max \left\{f_{i}, 0\right\}$ and $f_{i} \wedge 0=\max \left\{-f_{i}, 0\right\}$. We have the remaining cases when $f_{i} \geqslant 0$ or $f_{i} \leqslant 0$ for each $i$. In the following construction we assume $f_{i} \geqslant 0$ for each $i$, replacing $f_{i}$ by $-f_{i}$ if necessary. In this case, neither $f_{1} \geqslant f_{2}$ a.e. nor $f_{2} \geqslant f_{1}$ a.e. is true, otherwise, since $\left\|f_{1}\right\|=\left\|f_{2}\right\|, f_{1}=f_{2}$ a.e. Hence, neither $\left(f_{1}-f_{2}\right) \vee 0$ nor $\left(f_{1}-f_{2}\right) \wedge 0$ is equal to zero almost everywhere. The lemma is proved by letting $f_{1}^{*}=\left(\left(f_{1}-f_{2}\right) \vee 0\right) / \|\left(f_{1}-f_{2}\right)$ $\vee 0 \|$ and $f_{2}^{*}=\left(\left(f_{1}-f_{2}\right) \wedge 0\right) /\left\|\left(f_{1}-f_{2}\right) \wedge 0\right\|$.

LeMma 2.7. Let $\left\{f_{1}, \ldots, f_{m}\right\}$ be a subset of $F$ with disjoint supports and $\left\|f_{i}\right\|=1, f_{i} \geqslant u$ for all $1 \leqslant i \leqslant m$. If $f_{m+1}$ is linearly independent of 
$\left\{f_{1}, \ldots, f_{m}\right\}$ then there exists a set of nonnegative functions $\left\{f_{1}^{*}, \ldots, f_{m+1}^{*}\right\} \subset$ $F$ with disjoint supports and $\left\|f_{i}^{*}\right\|=1$ for $1 \leqslant i \leqslant m+1$.

Proof. Without loss of generality we may suppose $f_{m+1} \geqslant 0$ a.e. For if both $P\left(f_{m+1}\right)$ and $N\left(f_{m+1}\right)$ are nonempty, then either $f^{+}=f_{m+1} \vee 0$ or $f^{-}=f_{m+1} \wedge 0$ is linearly independent of $\left\{f_{1}, \ldots, f_{m}\right\}$. Otherwise,

$$
f^{+}=\sum_{i=1}^{m} a_{i} f_{i}, \quad f^{-}=\sum_{i=1}^{m} b_{i} f_{i}
$$

implies $f_{m+1}=f^{+}-f^{-}=\sum_{i=1}^{m}\left(a_{i}-b_{i}\right) f_{i}$ and $f_{m+1}$ is linearly dependent of $\left\{f_{1}, \ldots, f_{m}\right\}$.

Let $S_{1}=\cup_{i=1}^{m}$ spt $f_{i}$ and $S_{2}=\operatorname{spt} f_{m+1}$. Consider the following cases:

(1) $S_{1}$ and $S_{2}$ are disjoint. In this case the lemma is obvious. $f_{1}^{*}$ may be chosen as $f_{i}$ for $1 \leqslant i \leqslant m$ and $f_{m+1}^{*}=f_{m+1} /\left\|f_{m+1}\right\|$.

(2) $S_{1} \subset S_{2}$ and $S_{1} \neq S_{2}$. By repeated application of (2.6) and Lemma 2.5, $S_{2}-S_{1}$ is invariant. Hence, by Lemma $2.1, f_{m+1} \cdot X_{\left(S_{2}-S_{1}\right)}$ is invariant and the lemma holds for $f_{i}^{*}=f_{i}$ for all $1 \leqslant i \leqslant m$ and

$$
f_{m+1}^{*}=\left(f_{m+1} \cdot X_{\left(s_{2}-s_{1}\right)}\right) /\left\|f_{m+1} \cdot X_{\left(s_{2}-s_{1}\right)}\right\| \text {. }
$$

(3) $S_{2}-S_{1}=\varnothing$ and $S_{2} \subsetneq S_{1}$. There exists a function, say $f_{1}$, such that $A=\operatorname{spt} f_{1} \cap S_{2} \neq \varnothing$, and $f_{1} \neq f_{n+1}$ in $A$. Let $f_{1}^{\prime}=\left(f_{1} \cdot X_{A}\right) /\left\|f_{1} \cdot X_{A}\right\|$. By applying Lemma 2.6 , we have $f_{1}^{*}$ and $f_{n+1}^{*}$ with $\left\|f_{1}^{*}\right\|=\left\|f_{n+1}^{*}\right\|=1$ and disjoint supports. The results of the lemma follow by letting $f_{i}^{*}=f_{i}$ for all $2 \leqslant i \leqslant n$.

(4) $S_{1}=S_{2}$. Suppose for each $1 \leqslant i \leqslant m$, there exists $\alpha_{i} \neq 0$ such that $f_{n+1} \cdot X_{\text {spt } f_{i}}=\alpha_{i} f_{i}$. Then,

$$
f_{m+1}=\sum_{i=1}^{m} f_{m+1} \cdot X_{\text {spt } f_{i}}=\sum_{i=1}^{m} \alpha_{i} f_{i} .
$$

This is impossible since $f_{m+1}$ is independent of $\left\{f_{1}, \ldots, f_{m}\right\}$. Hence there exists a $j \in\{1, \ldots, m\}$ such that $f_{m+1} \cdot X_{\text {spt } f_{j}} /\left\|f_{m+1} \cdot X_{\text {spt } f_{j}}\right\|$ and $f_{j}$ are linearly independent functions with disjoint supports. By Lemma 2.6 there exists $f_{m+1}^{*} \geqslant 0$ and $f_{j}^{*} \geqslant 0$ in $F$ with disjoint supports and $\left\|f_{m+1}^{*}\right\|=\left\|f_{j}^{*}\right\|=$ 1 . Hence, the lemma is proved by choosing $f_{i}^{*}=f_{i}$ for $i \neq j$.

LEMMA 2.8. If $f \in F$ and spt $f=\cup_{k=0}^{P} I_{k}, 1 \leqslant P \leqslant \infty$, where all $I_{k}$ are closed intervals, then

(a) there exists $k_{0} \geqslant 0$ such that $I_{k_{0}}$ contains at least one discontinuity $x_{j}$, $j \in\{1, \ldots, k\}$ in its interior;

(b) $p<\infty$.

Proof. (a) Suppose for each $I_{k}, I_{k}$ does not contain any discontinuity $x_{j}$ in its interior. Choose any $k_{1}$ with $0 \leqslant k_{1} \leqslant p$. Then, since $I_{k_{1}}$ contains no $x_{j}$ in its interior, $\tau$ is strictly monotonic and continuous in $I_{k_{1}}$ with $\left|\tau^{\prime}\right|>1$. So, 


$$
\begin{aligned}
m\left(\tau\left(I_{k_{1}}\right)\right)>m\left(I_{k_{1}}\right) \text { and } \tau\left(I_{k_{1}}\right) & \neq I_{k_{1}} \text {. By Lemma 2.4, } \\
\qquad \bigcup_{k=0}^{P} \tau\left(I_{k}\right) & =\tau\left(\bigcup_{k=0}^{P} I_{k}\right)=\bigcup_{k=0}^{P} I_{k} \text { a.e. }
\end{aligned}
$$

Hence, $\tau\left(I_{k_{1}}\right) \subset \cup_{k=1}^{P} I_{k}$ a.e. On the other hand, $\tau\left(I_{k_{1}}\right)$ is an interval and the $I_{k}$ 's are disjoint. So, if $\tau\left(I_{k_{1}}\right) \cap I_{k_{2}} \neq \varnothing$ for some $k_{2} \neq k_{1}$ then $\tau\left(I_{k_{1}}\right)=I_{k_{2}}$ and $m\left(I_{k_{2}}\right)>m\left(I_{k_{1}}\right)$. By repeating the same argument, we may construct a sequence of $I_{k_{i}}$ 's with strictly increasing measures which are bounded below by $m\left(I_{k_{1}}\right)$. This. is impossible since all $I_{k}$ 's are disjoint and all of them are contained in finite interval $[0,1]$.

(b) By (a), there exists $k_{0} \geqslant 0$ such that $I_{k_{0}}$ contains at least one $x_{j}$ in its interior. Let

$$
D=\left\{k \in\{1, \ldots, P\}: I_{k} \text { contains } x_{j} \text { for some } j\right\} .
$$

Then $D$ is finite, since there are only finitely many points of discontinuities.

Let $r$ be such that $I_{r}$ is the shortest interval in the collection of intervals:

$$
\left\{\tau\left(I_{k}\right)\right\}_{k \in D} \cup\left\{I_{k}\right\}_{k \in D^{\circ}}
$$

Notice that $\left\{\tau\left(I_{k}\right)\right\}_{k \in D}$ consists of finite pieces of intervals. Let $S$ be the union of those intervals $I_{k}$ for which $m\left(I_{k}\right)>m\left(I_{r}\right)$. Then $S$ contains finitely many intervlas and it is obvious that $\tau(S) \subset S$ a.e. since if $m\left(I_{k}\right) \geqslant m\left(I_{r}\right)$ and $I_{k}$ does not contain any discontinuity $x_{j}$, then $m\left(\tau\left(I_{k}\right)\right)>m\left(I_{k}\right)>m\left(I_{r}\right)$. By Lemma 2.1,

$$
\int_{\tau^{-1}(S)-S} f=0
$$

Suppose there exists interval $K$ with $K \subset \operatorname{spt} f-S$. Choose $I_{1}$ to be the largest such interval. Since $K$ contains no discontinuity $x_{j}$, we have $\tau(K)$ is an interval of length greater than $m(K)$. Hence $\tau(K) \subset S$ and $K \subset \tau^{-1}(S)$. By (2.10), $\int_{K} f=0$. This is a contradiction since $K$ is in the support of $f$. Therefore $S=\operatorname{spt} f$ and $P<\infty$.

Proof of Theorem 1. By Lemmas 2.7 and 2.8, there exist at most $n$ functions in $F$ with disjoint supports and the support of each function contains at least one discontinuity $x_{j}$ in its interior. Let $H=\left\{f_{1}, \ldots, f_{n}\right\}$ be the set in $F$, which has the maximum number of functions having the above properties and $\left\|f_{i}\right\|=1$ for $f_{i} \in H$. Let $L_{i}=\operatorname{spt} f_{i}$. Then (1), (2), (3), (5) of Theorem 1 are satisfied. Suppose for some $i \in\{1, \ldots, n\}, f_{i}$ assumes both positive and negative values on subsets of $L_{i}$ with positive measures. Then, by letting $f_{i}^{1}=f_{i} \vee 0$ and $f_{i}^{2}=f_{i} \wedge 0$, we may increase the number of functions in $H$ by 1 . This is a contradiction. Hence, by replacing $f_{i}$ by $-f_{i}$ if necessary, we may assume $f_{i}>0$ on $L_{i}$ for all $1 \leqslant i \leqslant n$. In order to prove (6), let $g \in F$ satisfy (4), (5) for some $i \in\{1, \ldots, n\}$. If $g \neq f_{i}$ almost everywhere, then 
both $\left(g-f_{i}\right) \vee 0$ and $\left(g-f_{i}\right) \wedge 0$ are not equal to zero almost everywhere. This is impossible since $H$ has the maximum number of functions in $F$ having disjoint supports. Hence (6) is proved. If $f \in F$ and $f$ is linearly independent of $H$, then, by Lemma 2.7, we may again construct $n+1$ functions in $F$ with disjoint support. Hence $f=\sum_{i=1}^{n} a_{i} f_{i}$ with suitable choice of $a_{i}$ 's.

3. Let $\left\{L_{i}\right\}_{i=1}^{n}$ be the collection of sets stated in Theorem 1 . In this section we relate the limit set

$$
\Lambda(x)=\bigcap_{N=1}^{\infty} \overline{\left\{\tau^{n}(x)\right\}_{n=N}^{\infty}}
$$

to the sets $\left\{L_{i}\right\}$.

THEOREM 2. For almost every $x \in J, \Lambda(x)=L_{i}$ for some $i \in\{1, \ldots, n\}$.

Proof. Let

$$
\mathcal{E}_{i}=\bigcup_{k=0}^{\infty} \tau^{-k}\left(L_{i}\right)
$$

where $\tau^{-0}\left(L_{i}\right) \equiv L_{i}$. We first prove that $\cup_{i=1}^{n} \mathcal{L}_{i}=J$ almost everywhere. Suppose this is not the case. Then, there exists an interval $[a, b] \subset J-$ $\cup_{i=1}^{n} \mathcal{L}_{i}$. Let $f=X_{[a, b]}$. By a theorem of Lasota and Yorke [3],

$$
\frac{1}{m} \sum_{k=0}^{m-1} P_{\tau}^{k} f
$$

converges to a function $g \neq 0$ in the $L^{1}$ norm and $g$ is invariant under $\tau$. Let $L_{0}=$ spt $g$. Without loss of generality we may suppose $g>0$ in $L_{0}$. Then, $m\left(L_{i} \cap L_{0}\right)=0$ for $i=1, \ldots, n$. For, if $A \subset L_{i}$ for some $i \in\{1, \ldots, n\}$, then $\tau^{-k}(A) \subset \mathscr{L}_{i}$ for all $k=0,1 \ldots$ Hence,

$$
\int_{A} P_{\tau}^{k} f=\int_{A} P_{\tau^{k}} f=\int_{\tau^{-k}(A)} f=0
$$

for all $k=0,1 \ldots$ Therefore $\int_{A} g=0$, and $m\left(A \cap L_{i}\right)=0$. This is a contradiction to condition (7) of Theorem 1. Thus, we. have $J=\cup_{i=1}^{n} \mathfrak{L}_{i}$ a.e. Now, for almost every $x$ in $L_{i}$ by applying the Birkhoff Ergodic Theorem, we have

$$
\lim _{m \rightarrow \infty} \frac{1}{m} \sum_{k=0}^{m-1} X_{L}\left(\tau^{k}(x)\right)=\int_{L_{i}} f_{i}=1
$$

Hence, $\Lambda(x)=\bigcap_{N=1}^{\infty} \overline{\left\{\tau^{n}(x)\right\}_{n=N}^{\infty}} \subset L_{i}$. Since $\Lambda(x)$ is invariant under $\tau$, therefore $\Lambda(x)=L_{i}$. For, if not, $f_{i}$ restricted on $\Lambda(x)$ would be an invariant function which cannot be written as a linear combination of $\left\{f_{1} \ldots f_{n}\right\}$. 


\section{REFERENCES}

1. A. O. Gel'fond, $A$ common property of number systems, Izv. Akad. Nauk SSSR Ser. Mat. 23 (1959), 809-814. (Russian) MR 22 \#702.

2. A. Lasota, Invariant measures and functional equations, Aequationes Math. 9 (1973), 193-200. MR 48 \#6368.

3. A. Lasota and J. A. Yorke, On the existence of invariant measures for piecewise monotonic transformations, Trans. Amer. Math. Soc. 186 (1973), 481-488. (1974) MR 49 \# 538.

4. T. Y. Li and J. A. Yorke, Period three implies chaos, Amer. Math. Monthly 82 (1975), 985-992.

5. W. Parry, On the $\beta$-expansions of real numbers, Acta Math. Acad. Sci. Hungar. 11 (1960), 401-416. MR 26 \#288.

6. A. Rényi, Representation for real numbers and their ergodic properties, Acta Math. Acad. Sci. Hungar. 8 (1957), 477-493. MR 20 \#3843.

7. S. M. Ulam, $A$ collection of mathematical problems, Interscience Tracts in Pure and Appl. Math., no. 8, Interscience, New York, 1960. MR 22 \# 10884.

8. A. A. Kosjakin and E. A. Sandler, Ergodic properties of a certain class of piecewise smooth transformations of a segment, Izv. Vysšs. Učebn. Zaved. Matematika 1972, no. 3(118), 32-40. MR 45 \#8802.

Department of Mathematics, Michigan State University, East lansing, Michigan 48823

Institute for Fluid Dynamics and Applied Mathematics, University of Maryland, College Park, Maryland 20742 